\title{
CALCULATION OF DOSE, DOSE EQUIVALENT, AND RELATIVE BIOLOGICAL EFFECTIVENESS FOR HIGH CHARGE AND ENERGY ION BEAMS
}

\author{
J. W. Wilson, ${ }^{*}$ M. Reginatto ${ }^{\dagger}$ F. Hajnal, ${ }^{\dagger}$ and S. Y. Chun ${ }^{\ddagger}$
}

\begin{abstract}
The Green's function for the transport of ions of high charge and energy is utilized with a nuclear fragmentation database to evaluate dose, dose equivalent, and RBE for C3H10T1/2 cell survival and neoplastic transformation as a function of depth in soft tissue. Such evaluations are useful to estimates of biological risk for high altitude aircraft, space operations, accelerator operations, and biomedical applications.
\end{abstract}

Health Phys. 68(4):532-538; 1995

Key words: dose equivalent; relative biological effectiveness; dose; tissue, body

\section{INTRODUCTION}

The BIOLOGiCal response of living tissues depends (in part) on the temporal and spatial fluctuations of the energy deposits of the ions within the tissue system. Such fluctuations depend not only on the specific environment (or particle fluence) to which the person is exposed but also on how that environment (or particle fluence) is modified by interaction with the human body in reaching specific tissues. Only by knowledge of the specific radiation types and their physical properties at the tissue site can a basis for estimating risk be found. Even if the environment to which the individual is exposed is known precisely, the energy deposits within specific tissues deep in the body are largely known through theoretical estimates and are therefore limited by the uncertainty in the calculational models. Although methods for exposure evaluations in high energy nucleonic fields are relatively well developed (Alsmiller et al. 1970; Nabelssi and Hertel 1993), there are several practical applications in which exposures with high charge and energy (HZE) ions are of concern. Among these are the natural cosmic ray environment consisting of relativistic nuclei of all elements for which HZE ions contribute $80 \%$ of the free space dose equivalent (Wilson et al. 1991). Even for

* NASA Langley Research Center, Hampton, VA 23681-0001; $\ddagger$ DOE Environmental Measurements Laboratory, New York, NY 10014; Old Dominion University, Norfolk, VA 23508.

(Manuscript received 25 March 1994; revised manuscript received 5 September 1994, accepted 11 November 1994) $0017-9078 / 95 / \$ 3.00 / 0$

Copyright 1995 Health Physics Society modest depths of shield penetration $\left(30 \mathrm{~g} \mathrm{~cm}^{-2}\right)$, the HZE dose equivalent is on the order of $45 \%$ of the total and is of considerable importance to protection of astronauts and possible future high speed aircraft $(\approx$ Mach 2.5 or greater) on high latitude routes (for example, New York-London, London-Anchorage, Frankfort-Tokyo). Computational models are required for treatment planning in which cell killing for HZE tumor therapy is the critical parameter. With the further development of HZE accelerators, there is a growing concern among policy setting bodies on evaluation of exposures for the scattering of HZE particles from the beamline (L. Brackenbush $1994^{\S}$ ) for which the present calculations were undertaken. Clearly, an accurate conversion of the environment to estimates of exposure fields at specific tissue sites is an important issue in HZE radiation protection problems.

In our previous paper on the closed form solution for the HZE Green's function, we reviewed the computational procedures for space and laboratory HZE transport of various groups (Wilson et al. 1990). It is noted in that review that numerical solution methods for the Boltzmann equation are best suited to space radiations where energy spectra are smooth over large energy intervals and less suited to the simulation of laboratory beams which exhibit large spectral variation over a very limited energy domain and a large energy derivative resulting in large truncation errors (Wilson et al. 1990, 1991). In the present paper, we apply these analytical methods (Wilson et al. 1990) to evaluate dosimetric quantities as a function of penetration depth in tissue equivalent materials to estimate specific organ exposures.

\section{TRANSPORT EQUATION}

The Green's function is introduced as a solution of the following transport equation:

$$
\left[\frac{\partial}{\partial x}-\frac{\partial}{\partial E} \tilde{S}_{j}(E)+\sigma_{j}\right] G_{j m}\left(x, E, E^{\prime}\right)=\sum_{k} \sigma_{j k} G_{k m}\left(x, E, E^{\prime}\right),
$$

where $G_{j m}\left(x, E, E^{\prime}\right)$ is the flux of ion type $j$ at depth $x$ with energy $E$ (in $\mathrm{MeV} / \mathrm{amu}$ ) resulting from a unit flux of

\footnotetext{
\& Personal communication, L Brackenbush, Pacific Northwest Laboratory, POB999, Richland, WA 99352, 1994.
} 
type $m$ ions of energy $E^{\prime}$ at the boundary, $\tilde{S}_{j}(E)$ is the change in $E$ per unit distance, $\sigma_{j}$ is the total macroscopic reaction cross section, and $\sigma_{j k}$ the macroscopic cross section for collision of ion type $k$ to produce an ion of type $j$. The boundary condition is given by

$$
G_{j m}\left(0, E, E^{\prime}\right)=\delta_{j m} \delta\left(E-E^{\prime}\right),
$$

where $\delta_{j m}$ is Kronecker's delta and $\delta\left(E-E^{\prime}\right)$ is Dirac's delta. The dose and dose equivalent for monoenergetic ions are given by

$$
\begin{aligned}
& D_{m}\left(x, E^{\prime}\right)=\sum_{j} \int_{0}^{E} A_{j} \tilde{S}_{j}(E) G_{j m}\left(x, E, E^{\prime}\right) d E ; \text { and } \\
& H_{m}\left(x, E^{\prime}\right)=\sum_{j} \int_{0}^{E} A_{j} \tilde{S}_{j}(E) Q\left(L_{j}\right) G_{j m}\left(x, E, E^{\prime}\right) d E .
\end{aligned}
$$

If $G_{j m}\left(x, E, E^{\prime}\right)$ is known as a closed form solution (Wilson et al. 1990), then eqns (3) and (4) can be evaluated by simple integration techniques and the associated errors in numerically solving eqn (1) are avoided (Wilson et al. 1991).

The above equations can be simplified (Wilson 1977) by transforming the energy into the residual range of the ions as

$$
r_{j}=\int_{0}^{E} \frac{d e}{\tilde{S_{j}}(e)},
$$

and defining new field functions as

$$
\mathcal{G}_{j m}\left(x, r_{j}, r_{m}^{\prime}\right)=\tilde{S}_{j}(E) G_{j m}\left(x, E, E^{\prime}\right) .
$$

As a result, eqn (1) becomes

$$
\left(\frac{\partial}{\partial x}-\frac{\partial}{\partial r_{j}}+\sigma_{j}\right) G_{j m}\left(x, r_{j}, r_{m}^{\prime}\right)=\sum_{k} \frac{\nu_{j}}{\nu_{k}} \sigma_{j k} G_{k m}\left(x, r_{k}, r_{m}^{\prime}\right),
$$

with the boundary condition

$$
\varphi_{j m}\left(0, r_{j}, r_{m}^{\prime}\right)=\delta_{j m} \delta\left(r_{j}-r_{m}^{\prime}\right)
$$

Note that $\nu_{j}$ is the range scale factor such that $\nu_{j} r_{j}=$ $\nu_{m} r_{m}$ and can be expressed as

$$
\nu_{j}=Z_{j}^{2} / A_{j} .
$$

The solution to eqn (1) can be written as a perturbation series as

$$
\mathscr{G}_{j m}\left(x, r_{j}, r_{m}^{\prime}\right)=\sum_{i} G_{j m}^{(i)}\left(x, r_{j}, r_{m}^{\prime}\right)
$$

where

$$
\mathscr{G}_{j m}^{(0)}\left(x, r_{j}, r_{m}^{\prime}\right)=\exp \left(-\sigma_{j} x\right) \delta_{j m} \delta\left(x+r_{j}-r_{m}^{\prime}\right),
$$

and the first collision term is

$$
\boldsymbol{G}_{j m}^{\prime \prime \prime}\left(x, r_{j}, r_{m}^{\prime}\right)=\frac{\nu_{j} \sigma_{j m}}{\left|\nu_{m}-\nu_{j}\right|} \exp \left(-\sigma_{j} x_{j}-\sigma_{m} x_{m}\right),
$$

where $\mathscr{G}_{j m}^{(1)}\left(x, r_{j}, r_{m}^{\prime}\right)$ takes on non-zero values for

$$
\frac{\nu_{m}}{\nu_{j}}\left(r_{m}^{\prime}-x\right) \leq r_{j} \leq \frac{\nu_{m}}{\nu_{j}} r_{m}^{\prime}-x,
$$

and

$$
\begin{gathered}
x_{j}=\left(\nu_{m} x+\nu_{j} r_{j}-\nu_{m} r_{m}^{\prime}\right) /\left(\nu_{m}-\nu_{j}\right) ; \text { and } \\
x_{m}=\left(\nu_{m} r_{m}^{\prime}-\nu_{j} x-\nu_{j} r_{j}\right) /\left(\nu_{m}-\nu_{j}\right) .
\end{gathered}
$$

Note that in eqns (12)-(15), $x_{j}+x_{m}=x$ for all $r_{j}$ and $r_{m}^{\prime}$. The significance of $x_{m}$ is that it is the distance ion $m$ traveled from the boundary to the collision site at which the ion $j$ was produced and must now travel distance $x_{j}$ before reaching $x$. Higher order terms in the perturbation series are given in the Appendix.

To this point of development, the perturbative Green's function (eqn 10) can be approximated by summing the terms given by eqns (11), (A7), and (A9). The problem in explicitly summing eqn $(10)$ is that the higher order terms of eqn (A9) contain thousands of terms, each of which are composed of sums of tens of thousands of terms for the second order correction $(i=2)$ and millions of terms for the third order correction. Since the terms given by eqns (A7) and (A9) are dependent only on spatial coordinates, we seek an alternative means of summing the series (10) implicitly.

\section{NONPERTURBATIVE GREEN'S FUNCTION}

We now introduce nonperturbation terms for the summation in eqn (10). First we recall (Wilson et al. 1990 ) that the $g$-function of $n$ arguments is generated by the perturbation solution of the transport equation neglecting ionization energy loss given by

$$
\left(\begin{array}{l}
d \\
d x
\end{array}+\sigma_{j}\right) g_{j m}(x)=\sum_{k} \sigma_{j k} g_{k m}(x),
$$

subject to the boundary condition

$$
g_{j m}(0)=\delta_{j m}
$$

The solution is

$$
g_{j m}(x)=\delta_{j m g} g(m)+\sigma_{j m} g(j, m)+\sum_{k} \sigma_{j k} \sigma_{k m} g(j, k, m)+\ldots
$$

A term by term comparison of the series (18) with the series (10) shows that in the spectral average approximation (see Appendix) we may write

$$
\begin{aligned}
\mathscr{G}_{j m}\left(x, r_{j}, r_{m}^{\prime}\right) \approx \exp \left(-\sigma_{j} x\right) \delta_{j m} \delta\left(x+r_{j}-r_{m}^{\prime}\right) \\
+\frac{\nu_{j}\left[g_{j m}(x)-\exp \left(-\sigma_{j} x\right) \delta_{j m}\right]}{x\left(\nu_{m}-\nu_{j}\right)} .
\end{aligned}
$$


The advantage of eqn (19) is that $g_{j m}(x)$ satisfies the convolution product relation (Wilson et al. 1993a) as

$$
g_{j m}(x)=\sum_{k} g_{j k}(x-y) g_{k m}(y)
$$

for any positive values of $x$ and $y$. A solution for small $x$ is easily obtained from the first few terms of eqn (18). Eqn (20) may then be used to propagate the function $g_{j m}(x)$ over the entire solution domain taking arbitrarily large steps. Although the nonperturbation Green's function in the form of eqns (19) and (20) provides a rapid computational method, the spectral terms are replaced by averages over the spectral domains of higher order terms. These energy averaged spectra can be corrected with the spectral distributions of perturbation theory. The first collision Green's function is given as

$$
\mathscr{G}_{j m}^{\prime \prime)}\left(x, r_{j}, r_{m}^{\prime}\right)=\frac{\nu_{j} \sigma_{j m}}{\left|\nu_{m}-\nu_{j}\right|} \exp \left(-\sigma_{j} x_{j}-\sigma_{m} x_{m}\right)
$$

We rewrite $\mathcal{G}_{j m}^{(1)}$ in terms of its average value (given by eqn A7) as

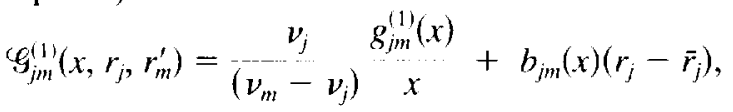

where $g_{j m}^{(1)}(x)=\sigma_{j m} g(j, m)$ is given by eqn (A7) and

$$
\bar{r}_{j}=\frac{r_{j l}+r_{j u}}{2}
$$

is the midpoint $\bar{r}_{j}$ between its limits given by eqn (A8). The $b_{j m}$ term of eqn (22) has the property that

$$
\int_{r_{j i}}^{r_{j u}} b_{j m}(x)\left(r-\bar{r}_{j}\right) d r=0
$$

to insure that the first term of eqn (22) is indeed the average spectrum as required. The spectral slope parameter is

$$
b_{j m}(x)=\frac{\nu_{j}^{2} \sigma_{j m}\left[\exp \left(-\sigma_{j} x\right)-\exp \left(-\sigma_{m} x\right)\right]}{x\left(\nu_{m}-\nu_{j}\right)\left|\nu_{m}-\nu_{j}\right|} .
$$

Note that the spectral slope results from the difference in mean free paths of the projectile and secondary ion. The ion $j$ at $x$ produced near $x_{m}=0$ is less attenuated than those produced locally $\left(x_{m}=x\right)$. Since those produced locally are generally of lower energy than those produced at $x=0$ we obtain the spectral slope.

\section{DEPTH-DOSE RELATIONS}

We consider the evaluation of dose equivalent as given by eqn (4) using the present formalism. The quality factor chosen is the latest recommended values of the ICRP (1990) given by

$$
Q(L)=\left\{\begin{array}{cc}
1 & \mathrm{~L}<10 \mathrm{keV} / \mu \mathrm{m} \\
0.32 \mathrm{~L}-2.2 & 10 \leq \mathrm{L} \leq 100 \mathrm{keV} / \mu \mathrm{m} \\
300 / \sqrt[\mathrm{L}]{ } & \mathrm{L}>100 \mathrm{keV} / \mu \mathrm{m}
\end{array}\right.
$$

April 1995, Volume 68, Number 4

It is clear from the above formalism that

$$
\begin{aligned}
H_{m}\left(x, E^{\prime}\right)= & Q\left[L_{m}\left(E_{0}\right)\right] A_{m} \tilde{S}_{m}\left(E_{0}\right) e^{-\sigma_{m} x} \\
& +\sum_{j} A_{j} \frac{\nu_{j}\left[g_{j m}(x)-\delta_{j m} \exp \left(-\sigma_{m} x\right)\right]}{\left(\nu_{m}-\nu_{j}\right) x} \\
& \times \int_{E_{j \prime}}^{E_{j u}} Q\left[L_{j}(E)\right] d E \\
& +\sum_{j} A_{j} b_{j m}(x) \int_{E_{j l}}^{E_{j u}} Q\left[L_{j}(E)\right]\left[R_{j}(E)-\bar{r}_{j}\right] d E
\end{aligned}
$$

where $E_{j u}$ and $E_{j l}$ are obtained from the limits in eqn (13). $D_{m}\left(x, E^{\prime}\right)$ is similarly obtained from eqn (26) by setting $Q(L) \Rightarrow 1$.

The ion flux within a water column is represented by the eighty most important isotopes lighter than ${ }^{59} \mathrm{Ni}$. The Green's function contains 6,400 terms as related by 3,160 fragmentation cross sections. The ion flux within the column, due to a monoenergetic incident ion flux, is evaluated using the NUCFRG database (Wilson et al. 1987; Townsend et al. 1993; Wilson et al. 1994) for the oxygen constituents and the Silberberg-Tsao (Silberberg et al.1976) model for the hydrogen constituents. The isobaric nuclear fragmentation cross sections are shown in Fig. 1. The nuclear absorption cross sections are shown in Fig. 2 for selected ions. In the following calculations, the nuclear cross sections were evaluated at the ion beam energy at the entrance to the water column. The results of the computations using eqn (26) with $Q \Rightarrow$ 1 for $D_{m}\left(x, E^{\prime}\right)$ are shown in Fig. 3 for ${ }^{20} \mathrm{Ne}$ beams and ${ }^{40} \mathrm{Ar}$ beams in a water column with the experimental measurements of Schimmerling et al. (1989) and Lyman
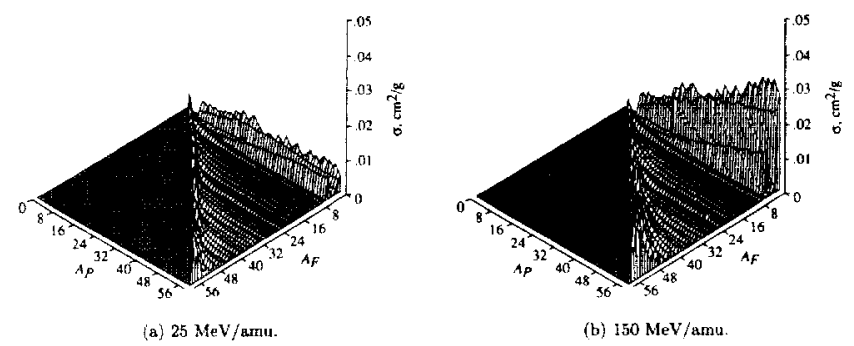

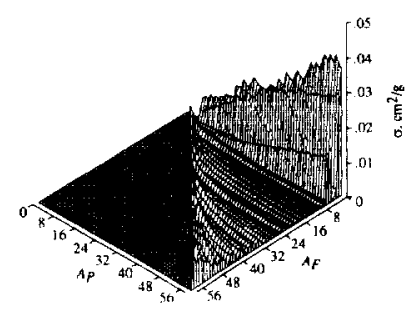

c) $600 \mathrm{MeV} / \mathrm{smu}$



(d) $2400 \mathrm{MeV} / \mathrm{amu}$.
Fig. 1. Isobaric fragmentation cross sections projectiles of ${ }^{6} \mathrm{Li}$ to ${ }^{59} \mathrm{Ni}$ for four incident energies in water targets. 


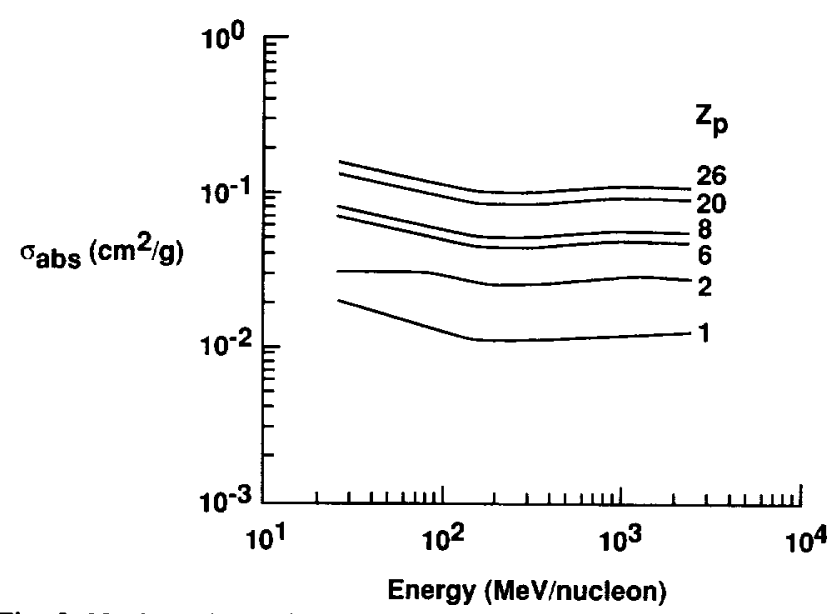

Fig. 2. Nuclear absorption cross sections of selected projectiles in water.

et al. (1977). Reasonable agreement is obtained in each case although there are important differences. These differences are on the order of $20 \%$ or less and are consistent with the comparison of LET spectral measurements of specific fragments (Shavers et al. 1993) measured in the ${ }^{20} \mathrm{Ne}$ experiments. Improvement must come through more accurate nuclear models, addition of energy dependence in the nuclear cross sections, and contributions from target breakup.

The average quality factors $H_{m}\left(x, E^{\prime}\right) / D_{m}\left(x, E^{\prime}\right)$ for fixed range for beams of ${ }^{20} \mathrm{Ne},{ }^{40} \mathrm{Ar}$, and ${ }^{56} \mathrm{Fe}$ are shown in Fig. 4. The quality factor is relatively constant over the range of the primary beam except in the Bragg peak region. Beyond the Bragg peak the average quality factor for ${ }^{20} \mathrm{Ne}$ beams drops suddenly as expected and declines slowly to larger depths since only the lighter ions penetrate to large depths. The depression of the average quality factor at the Bragg peak of the ${ }^{40} \mathrm{Ar}$ and ${ }^{56} \mathrm{Fe}$ beams is due to the decline of the primary ion quality factor $(Q \approx 300 / \sqrt{L})$ and the rapid rise in the primary ion dose at the Bragg peak.

There is experimental evidence that relative biological effectiveness depends not only on the rate at which an ion gives up energy to the tissue medium (numerically equal to LET) but depends as well on the lateral extent of the energy deposit (track structure effects). For this reason we consider a track structure cellular repair model for survival and neoplastic transformation (Wilson et al. 1991) as applied to the C3H10T1/2 mouse embryo cell culture for which there are extensive data using HZE ion beams (Yang et al. 1985, 1989). The cellular repair model is derived from the following assumptions. Injury from gamma rays follows Poisson statistics with a characteristic dose $D_{0}$, and enzymatic repair is less efficient with increasing number of hits. Such a model is consistent with the concept of a high efficiency and fast but saturable repair enzyme pool in competition with a slower less efficient repair process (Tubiana et al. 1990). Injury within a particle track is mediated by the secondary electrons (delta rays) and is related to the gamma-ray

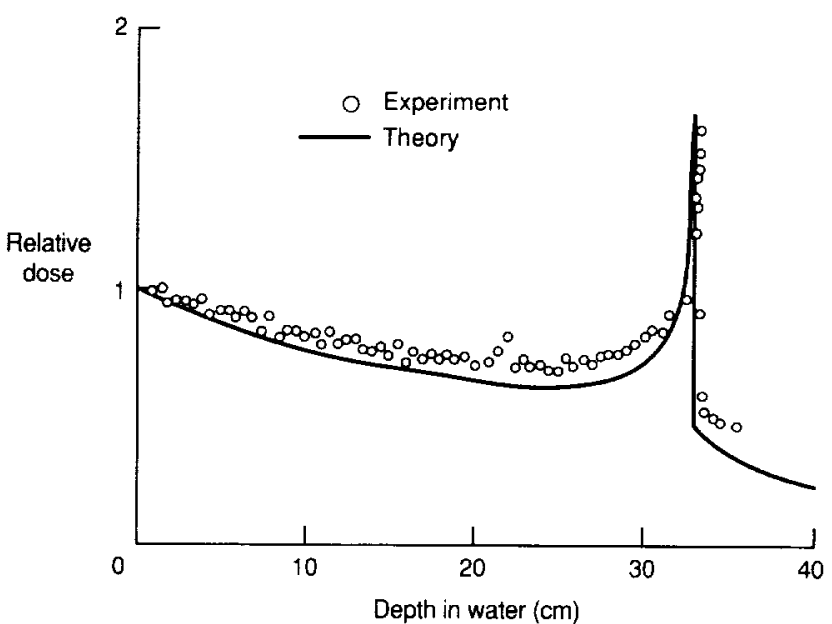

(a) Neon

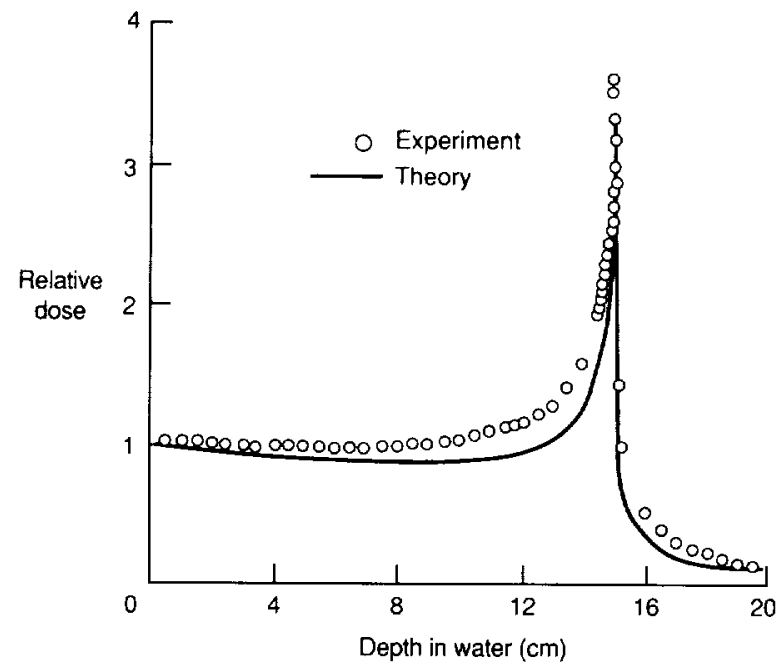

(b) Argon

Fig. 3. Bragg curves for (a) $\mathrm{Ne}$ and (b) Ar ion beam according to present calculations and experiments.

injury through $D_{0}$. An 'inactivation' cross section associated with the core of the ion track is approximately given by

$$
\sigma=\sigma_{0}\left(1-e^{\left.-Z^{\prime 2} / \kappa \beta^{2}\right)^{m},}\right.
$$

where $\kappa$ is a nondimensional size parameter associated with the sensitive structure within the cell, $Z^{\prime}$ is the ion effective charge, and $\beta$ is the ion velocity in units of the velocity of light (Katz et al. 1971). In the track periphery, the injury level is below saturation and multihit repair kinetics as in the case of gamma-ray exposures are assumed and the probability of direct inactivation is given as $p=\sigma / \sigma_{0}$ where $\sigma_{0}$ is the Katz 'saturation' cross section. The probability of injury with multihit repair kinetics is $(1-P)$. In the low dose rate limit (Wilson et al. 1993b), the nonsurviving fraction of exposed cells is

$$
n_{m}(t) \approx \frac{\alpha_{m 1}}{n_{1}} 6^{1 / 3}{ }^{(1-P) D} D_{o}+{ }_{L} D
$$




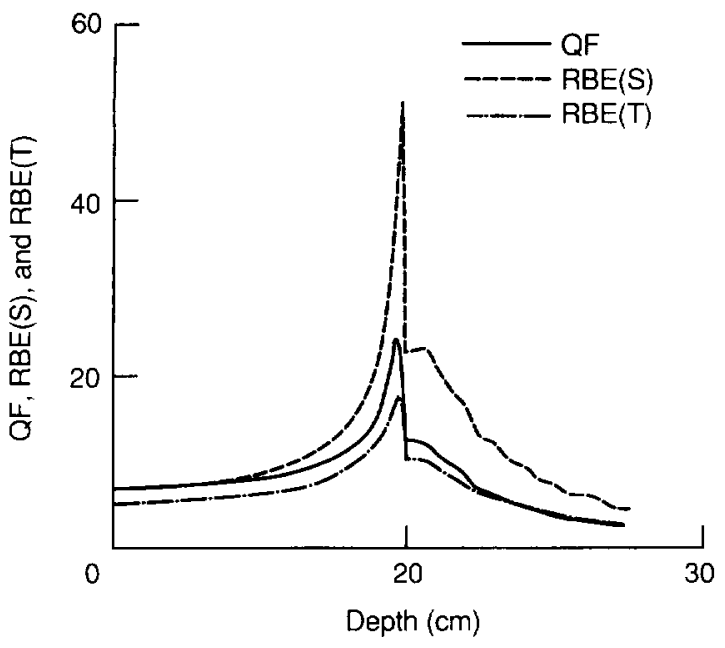

(a) Neon (454A MeV)

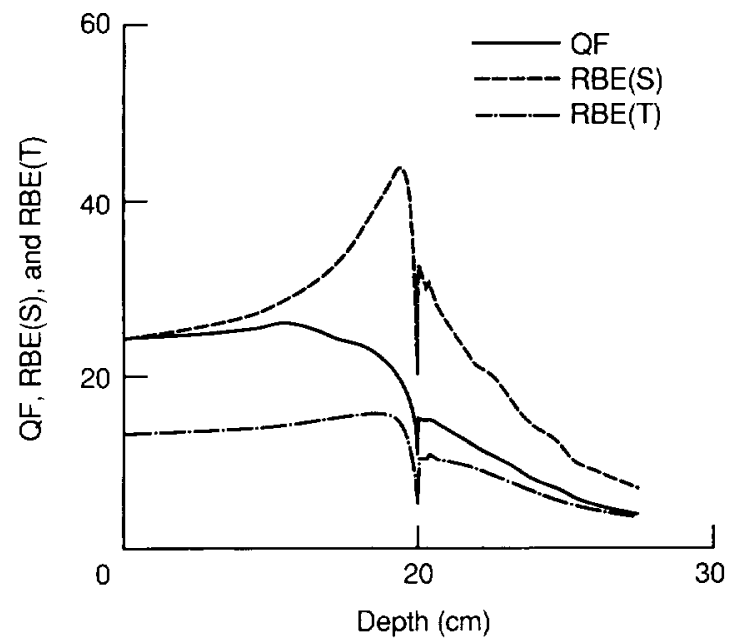

(b) Argon (620A MeV)

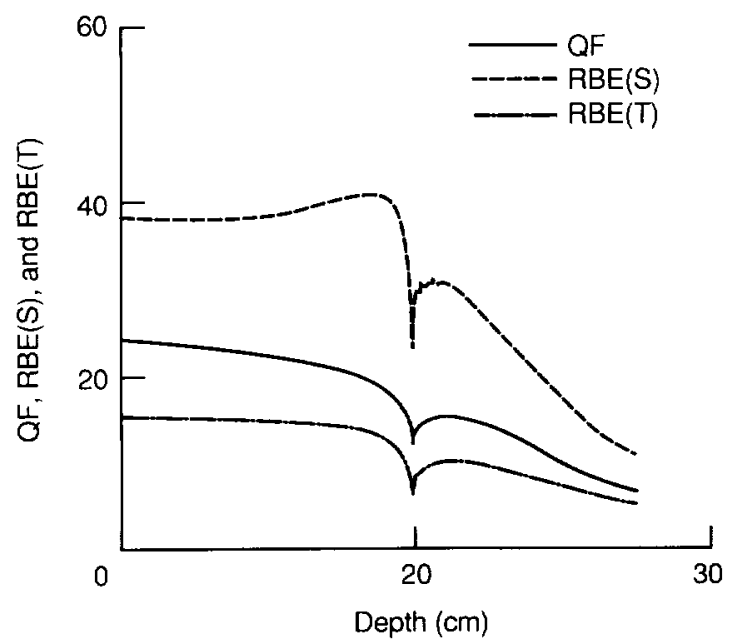

Fig. 4. Average quality factor and $\mathrm{RBE}_{m}$ values for $\mathrm{C} 3 \mathrm{H} 101 / 2$ cell survival (S) and transformation (T) for ions of $20 \mathrm{~cm}$ range in water; (a) ${ }^{20} \mathrm{Ne}$, (b) ${ }^{40} \mathrm{Ar}$, (c) ${ }^{56} \mathrm{Fe}$. where $D$ is the accumulated dose, $L$ is the linear energy transfer, $\alpha_{m 1}$ is the misrepair rate for once hit cells, $\alpha_{1}$ is the total rate at which the enzyme $(Z)$ forms a repair complex $\left(C^{\prime}\right)$ and completes the repair through the reaction kinetics given by

$$
\mathrm{Z}+\mathrm{DNA}_{l} \rightarrow C^{\prime} \searrow \begin{array}{ll}
\nearrow & \mathrm{Z}+\mathrm{DNA} \\
\mathrm{Z}+\mathrm{DNA}_{m}
\end{array}
$$

where subscript $I$ denotes injured DNA and subscript $m$ denotes misrepair. Note that $\left(1-\alpha_{m 1} / \alpha_{1}\right)$ and $\alpha_{m 1} / \alpha_{1}$ are the branching ratios of the reaction to perfectly repaired and misrepaired states. In eqn $(28)$, the $6^{1 / 3}(1-P) D / D_{0}$ is the fraction of cells with injury in the track periphery and $\alpha_{m 1} / \alpha$ is the probability the cell is permanently harmed. The fraction of cells exposed within the ion core with no chance of recovery is $\sigma D / L$. A similar result holds for the fraction of transformed cells in the low dose rate limit with appropriate parameters for transformation. The repair rates and efficiencies (which depend on the cell cycle status) are found from the experiments of Yang et al. $(1985,1989)$ and the low dose rate limit parameters for resting $G_{0}$ phase and exponential growth phase cells are given in Table 1 . Unlike conventional dosimetric analysis wherein radiation quality is represented by LET dependent factors, the repair kinetics model is driven by track-structure dependent injury coefficients (eqn 27). The $\mathrm{RBE}_{m}$ is found by finding the gamma-ray dose and ion dose which leads to the same fraction of changed cells (note $P=0$ for gamma rays) as

$$
\mathrm{RBE}_{m}=1-P+\frac{\alpha_{1}}{\alpha_{m 1}} \frac{\sigma D_{0}}{36 L} .
$$

The subscript $m$ on RBE denotes the maximum value obtained in the low dose rate limit. The $\mathrm{RBE}_{m}$ values derived from a cell kinetics model (Wilson et al. 1993b) for $\mathrm{C} 3 \mathrm{H} 10 \mathrm{~T} 1 / 2$ cells exposed and repaired in stationary phase at the low dose rate limit are substituted for $Q(L)$ in eqn (27) for ion beam exposures. The $\mathrm{RBE}_{m}$ for cell survival (S) and cell neoplastic transformation (T) are shown in comparison to the average quality factors. The quality factor is intended to represent stochastic processes (mainly cancer induction) and should best correlate with the RBE for neoplastic transformed cells (a pre-cancerous cell). The RBE for cell survival is more indicative of deterministic effects for which large RBEs

\begin{tabular}{|c|c|c|c|c|}
\hline \multicolumn{5}{|c|}{ Cellular kinetic ratio $\alpha_{m_{1}} \alpha_{1}-1$} \\
\hline & \multicolumn{2}{|l|}{$G_{0}$ phase } & \multicolumn{2}{|c|}{ Exponential phase } \\
\hline $\begin{array}{l}\text { Survival } \\
\text { Transformation }\end{array}$ & $\begin{array}{l}\approx 0.03 \\
\approx .002\end{array}$ & & \multicolumn{2}{|c|}{$\begin{array}{r}0.30 \\
.01\end{array}$} \\
\hline \multicolumn{5}{|c|}{ Katz C3H10T1/2 cell parameters } \\
\hline & $\sigma_{0}, \mathrm{~cm}^{2}$ & $\kappa$ & $m$ & $D_{01}, \mathrm{~Gy}$ \\
\hline Survival & $5 \times 10^{-7}$ & 750 & 3 & 2.8 \\
\hline
\end{tabular}

Table 1. Cellular kinetic ratios and parameters. 
have been measured for mouse embryo hemopoiesis (Jiang et al. 1994) irradiated with high LET alpha particles. Many of the qualitative features of the $\mathrm{RBE}_{m}$ are represented by the average quality factor but important track structure dependent differences can be observed. The quality factor increases from beam entrance to the position at which the primary ion reaches 100 $\mathrm{keV} / \mu \mathrm{m}$ and declines to greater depth as seen for neon and argon ions. The iron ions enter with LET $>100$ $\mathrm{keV} / \mu \mathrm{m}$ and the quality factor declines throughout the medium. The quality factor approaches the stochastic $\mathrm{RBE}(\mathrm{T})$ for the lighter more penetrating fragments downstream from the Bragg peak. Near beam entrance, the width of the primary track is maximum and the RBE(T) lies substantially below the LET dependent quality factor. It is accidental that $\operatorname{RBE}(\mathrm{S})$ coincides with quality factor at beam entrance for neon and argon. The diverse behavior of $\operatorname{RBE}(\mathrm{S})$ and $\operatorname{RBE}(\mathrm{T})$ is a result of the specific track structure parameters associated with survival and transformation (Table 1) and are consistent with the data of Yang et al. (Wilson et al. 1993b). The quality factor is most appropriate for representing the cell transformation $\mathrm{RBE}_{m}$ for lighter ions such as ${ }^{20} \mathrm{Ne}$ and appears to be conservative for heavier ions. A quality factor for deterministic effects is not defined but would be more indicative of $\operatorname{RBE}(\mathrm{S})$. Note that the present result neglects the secondaries produced from tissue constituents (target fragments).

\section{CONCLUSIONS}

At this point of development of HZE transport theory, the fluence spectra of the multiple charged ions can be reasonably calculated in one dimension assuming constant nuclear cross sections. Clearly, future efforts should concentrate on deriving important corrections associated with realistic cross section variation with energy. Even with this improvement several issues remain in comparing the results to the experimental data. The target breakup will make contributions at all depths but may be a source of error near the Bragg region and beyond and especially for the lighter ion beams. Beyond this, significant improvements are required in the nuclear models used in generating the nuclear database.

\section{REFERENCES}

Alsmiller, R. G.; Armstrong, T. W.; Coleman, W. A. The absorbed dose and dose equivalent from neutrons in the energy range 60 to $3000 \mathrm{MeV}$ and protons in the energy range 400 to $3000 \mathrm{MeV}$. Nuclear Sci, and Eng. 42:367-381; 1970.

Jiang, T. N.; Lord, B. I.; Hendry, J. H. Alpha particles are extremely damaging to developing hemopoiesis compared to gamma irradiation. Radiat. Res. 137:380-384; 1994.

Katz, R.; Ackerson, B.; Homayoonfar, M.; Sharma, S. G. Inactivation of cells by heavy Ion bombardment. Radiat. Res. 47:402-425;1971.

Lyman, J. T.; Howard, J. Dosimetry and instrumentation for helium and heavy ions. Radiat. Oncology Bio. Phys. 3:81$85 ; 1977$.
Nabelssi, B. K.; Hertel, N. E. Effective dose equivalents and effective doses for neutrons from 30 to $180 \mathrm{MeV}$. Radiat. Prot. Dosim. 48:227-243; 1993.

Schimmerling, W.; Miller, J.; Wong, M.; Rapkin, M.; Howard, J.; Spieler, H. G. S.; Jarret, B. V. The fragmentation of $670 \mathrm{~A} \mathrm{MeV} \mathrm{Neon-20}$ as a function of depth in water. I. experiment. Radiat Res. 120:36-71;1989.

Shavers, M. R.; Frankel, K.; Miller, J.; Schimmerling, W.; Townsend, L. W.; Wilson, J. W. The fragmentation of $670 \mathrm{~A}$ MeV Neon-20 as a function of depth in water. III. Analytic multi-generation transport theory. Radiat. Res. 136:1-14; 1993.

Silberberg, R. C.; Tsao, H.; Shapiro, M. M. Semiempirical cross sections and applications to nuclear interactions of cosmic rays. In: Shen, B. S. P.; Merker, M., eds. Spallation nuclear reactions and their applications. Dordrecht-Holland: D. Reidel Publishing Company;1976:49-81.

Townsend, L. W.; Wilson, J. W.; Tripathi, R. K.; Norbury, J. W.; Badavi, F. F.; Khan, F. HZEFRG1; An energy dependent semiempirical nuclear fragmentation model. Washington, D.C.: NASA TP-3310; 1993.

Tubiana, M.; Dutreix, J.; Wambersie, A. Introduction to radiobiology. London: Taylor and Francis; 1990:51-57.

Wilson, J. W. Analysis of the theory of high-energy ion transport. Washington, D.C.: NASA TN D-8381, 1977.

Wilson, J. W.; Badavi, F. F. New directions in heavy ion shielding. In: Proceedings of the Topical Meeting on New Horizons in Radiation Protection and Shielding. American Nuclear Society, Inc.; 1992:198-202.

Wilson, J. W.; Costen, R. C.; Shinn, J. L.; Badavi, F. F. Green's function methods in heavy ion shielding. Washington, D.C.: NASA TP-3311; 1993a.

Wilson, J. W.; Cucinotta, F. A.; Shinn, J. L. Cell kinetics and track structure. In: Swenberg, C. E.; Horneck, G.; Stassinopoulos, E. G., eds. Biological effects and physics of solar and galactic cosmic radiation. New York: Plenum Press; 1993b:295-338.

Wilson, J. W.; Lamkin, S. L; Farhat, H.; Ganapol, B. D.; Townsend, L. W. A closed form solution to HZE propagation. Radiat. Res. 122:223-228; 1990.

Wilson, J. W.; Shinn, J. L.; Townsend, L. W.; Tripathi, R. K.; Badavi, F. F.; Chun, S. Y. NUCFRG2: A semiempirical nuclear fragmentation model. Nucl. Inst. Methods. Phys. Res. B94:95-102; 1994.

Wilson, J. W; Townsend, L. W.; Badavi, F. F. A semiempirical nuclear fragmentation model. Nucl. Inst. and Methods Phys. Res. B18:225-231; 1987.

Wilson, J. W.; Townsend, L. W.; Bidasaria, H. B.; Schimmerling, W.; Wong, M.; Howard, J. ${ }^{20} \mathrm{Ne}$ depth-dose relations in water. Health Phys. 46:1101-1111; 1984.

Wilson, J. W.; Townsend, L. W.; Schimmerling, W.; Khandelwal, G. S.; Khan, F.; Nealy, J. E.; Cucinotta, F. A.; Simonsen, L. C.; Shinn, J. L.; Norbury, J. W. Transport methods and interactions for space radiations. Washington, D.C.: NASA Reference Publication 1257;1991.

Yang, T. C.; Craise, L. M.; Mei, M. T.; Tobias, C. A. Neoplastic cell transformation by heavy charged particles. Radiat. Res. 104:5177-5187; 1985.

Yang, T. C.; Craise, L. M.; Mei, M. T.; Tobias, C. A. Neoplastic cell transformation by high-LET radiation: Molecular mechanisms. Adv. Sp. Res. 9:131-140; 1989. 
APPENDIX

The second collision term is similarly

$$
\begin{aligned}
\mathscr{G}_{j m}^{(2)}\left(x, r_{j}, r_{m,}^{\prime}\right) & =\sum_{k} \begin{array}{c}
\nu_{j} \sigma_{j k} \sigma_{k m} \\
\left|\nu_{m}-\nu_{k}\right|
\end{array} \\
& \times \int_{x_{i /}}^{i_{m}} \exp \left(-\sigma_{j} x_{j}-\sigma_{k} x_{k}-\sigma_{m} x_{m}\right) d x_{j},
\end{aligned}
$$

where

$$
\begin{gathered}
x_{j}+x_{k}+x_{m}=x \\
\nu_{j} x_{j}+\nu_{k} x_{k}+\nu_{m} x_{m}=\nu_{m} r_{m}^{\prime}-\nu_{k} r_{k},
\end{gathered}
$$

and the integral limits in eqn (A1) depend on $\nu_{m}, \nu_{k}, \nu_{j}$ and the physical requirements that the distances $x_{j}, x_{k}$, and $x_{m}$ all be positive. We have used the rather weak dependence of the exponential argument of eqns (12) and (A1) on energy to simplify spectral quantities in the past (Wilson et al. 1984). Higher order terms are similarly derived (Wilson et al. 1990). Whenever $r_{i}, r_{m}^{\prime}>x$ it can be shown that eqn (12) has the property that

$$
\int_{r_{j}}^{r_{m}} \mathscr{G}_{j m}\left(x, r_{j}, r_{m}^{\prime}\right) d r_{j}=\sigma_{j m} g(j, m),
$$

where $g(j, m)$ is the $g$-function of two arguments introduced in our prior paper (Wilson et al. 1990) and the integral limits are the interval limits defined by eqn (13). The $g$-functions are given by the following relations

$$
g(j)=\exp \left(-\sigma_{j} x\right)
$$

and

$$
\begin{aligned}
& g\left(j_{1}, j_{2}, \ldots, j_{n}, j_{n+1}\right) \\
& =\frac{g\left(j_{1}, j_{2}, \ldots, j_{n-1}, j_{n}\right)-g\left(j_{1}, j_{2}, \ldots, j_{n} \quad, j_{n+1}\right) .}{\sigma_{j_{n+1}}-\sigma_{j_{n}}} .
\end{aligned}
$$

The first collision Green's function term may be approximated by the spectral averaged value as

$$
\begin{aligned}
\mathscr{G}_{j m}^{(1)}\left(x, r_{j}, r_{m}^{\prime}\right) & \approx \frac{1}{r_{j u}-r_{j l}} \int_{r_{r} t}^{r_{j u}} \mathcal{G}_{j m}^{(1)}\left(x, r_{j}, r_{m}^{\prime}\right) d r_{j} \\
& =\frac{\nu_{j} \sigma_{j m} g(j, m)}{x\left(\nu_{m}-\nu_{j}\right)}
\end{aligned}
$$

and is functionally dependent only on depth of penetration $x$ (Wilson and Badavi 1992). Its contribution applies only over the energy range

$$
{ }_{\nu_{m}}^{\nu_{j}}\left(r_{j}+x\right) \leq r_{m}^{\prime} \leq{\stackrel{\nu}{\nu_{m}}}_{\nu_{m}} r_{j}+x
$$

for $\nu_{m}>\nu_{j}$. If $\nu_{j}>\nu_{m}$, as can happen in neutron removal collisions, the negative of eqn (A7) is used and the inequalities of eqn (A8) are reversed (Wilson et al. 1990). The higher order terms are approximated (Wilson and Badavi 1992) by their spectral averages as

$$
\begin{aligned}
& \mathscr{G}_{j m}^{(i)}\left(x, r_{j}, r_{m}^{\prime}\right) \\
& \quad \approx \sum_{\left.k_{1}, k_{2}, \ldots, k_{i}\right)} \nu_{j} \sigma_{j k_{1}} \sigma_{k_{1} k_{2}} \cdots \sigma_{k_{i}}, m g\left(j, k_{1}, k_{2}, \cdots, k_{i-1}, m\right) \\
& x\left(\nu_{m}-\nu_{j}\right)
\end{aligned}
$$

Note that $\varphi_{j m}^{(i)}\left(x, r_{j}, r_{m}^{\prime}\right)$ is purely dependent on $x$ for $i>$ 0 , and we represent the expression as $\mathscr{G}_{j m}^{(i)}(x)$. 
